\title{
The Full Brownian Web as Scaling Limit of Stochastic Flows
}

\author{
L. R. G. Fontes $\quad$ C. M. Newman
}

\begin{abstract}
In this paper we construct an object which we call the full Brownian web (FBW) and prove that the collection of all space-time trajectories of a class of one-dimensional stochastic flows converges weakly, under diffusive rescaling, to the FBW. The (forward) paths of the FBW include the coalescing Brownian motions of the ordinary Brownian web along with bifurcating paths. Convergence of rescaled stochastic flows to the FBW follows from general characterization and convergence theorems that we present here combined with earlier results of Piterbarg.
\end{abstract}

Keywords and Phrases: Stochastic flows, Scaling limit, Brownian web, Full Brownian web, Coalescing Brownian motions, Expansions and contractions AMS 2000 Subject Classifications: 60B05, 60B10, 60B12, 60G17, 60J30, $60 \mathrm{~J} 65$

\section{Introduction}

In studying certain one-dimensional stochastic flows [5], Piterbarg [6] 7] showed that on the one hand their (diffusive) scaling limits led to coalescing Brownian motions while on the other hand there were necessarily regions of space time where expansions of trajectories occur rather than the contractions corresponding to coalescing. Regarding a stochastic flow as the collection of all its space-time trajectories (or paths with time $t \in(-\infty, \infty)$ ), Piterbarg's results suggested to us that the scaling limit should be expressible as a collection of paths in space-time related to the Brownian web [1, 9, 2, 3] but, unlike the Brownian web, including bifurcation as well as coalescence. 
One main result of this paper (see Theorem 5.1) is a proof that the scaling limit of the collection of all stochastic flow paths is indeed such an object, which we call the full Brownian web (FBW). Of course, to prove such a theorem, it is useful to have first defined the (putative) limiting object and this is our other main result, the construction (see Section 2) of the FBW.

The other crucial results of this paper are a characterization, given in Section 3, of the FBW and a general convergence theorem (see Theorem 4.1) based on this characterization. Theorem 5.1 giving weak convergence of the rescaled stochastic flow to the FBW is then an immediate consequence of our general convergence theorem combined with Piterbarg's earlier results [6, 7]. We note that we only need Piterbarg's result (Theorem 6 in [7]) concerning convergence of finite dimensional distributions of forward paths to coalescing Brownian motions rather than his stronger result (see Theorem 1 in [7]) about weak convergence of the rescaled forward stochastic flow in the Skorohod topology. We expect that Piterbarg's stronger convergence can be obtained as a consequence of Theorem 5.1 below, but have not examined this in detail. We conclude the introduction with more details about the FBW.

The FBW is a collection of continuous noncrossing paths from $\mathbb{R}$ to $\mathbb{R}$ (which may nonetheless touch, coalesce with or bifurcate from each other) with the following properties. From every deterministic space-time point $(x, t)$, there is almost surely a unique (doubly infinite; i.e., defined for $-\infty<$ $t<\infty)$ path through that point. We will use the term semipath to denote both the forward in time semi-infinite path starting at $(x, t)$ and defined for times $s \geq t$ and the backward in time semi-infinite path ending at $(x, t)$. The semipath from $(x, t)$ is distributed as a Brownian path (with unit diffusion coefficient) starting at that point, and the backward semipath from $(x, t)$ is distributed as a backward Brownian path (with unit diffusion coefficient) from that point. Furthermore, for every deterministic finite set of space-time points, the forward semipaths starting from those points are distributed as coalescing Brownian paths (with unit diffusion coefficient) starting from those points; an analogous statement holds for the backward semipaths.

As already mentioned, the FBW arises as the scaling limit of certain stochastic flows of homeomorphisms [5, 7], as defined in Section [5, and can be constructed as a functional of the (double) Brownian web 2, 3, 4] (see also [9]). The structure of the FBW sheds some light on and also raises some questions about the nature of contracting and expanding (space-time) regions for these stochastic flows beyond Piterbarg's analysis in [7]. Here are three examples. 
1. According to Proposition 2.5] below (see also Remark 2.7), every nontrivial path $\gamma$ in the FBW consists of a backward Brownian path and a forward Brownian path spliced together at some $\left(x^{*}, t^{*}\right)$. The backward path should be regarded as passing through a space-time region of expansion, since every point $(x, t)$ with $t<t^{*}$ along it has the property that an FBW path through $\left(x_{1}, t\right)$ for $x_{1}<x$ does not coalesce with one through $\left(x_{2}, t\right)$ for $x_{2}>x$ until some time after $t^{*}$, no matter how close $x_{1}$ and $x_{2}$ are to $x$ and each other. On the other hand the forward path should be regarded as passing through an essentially contractive region since for generic points $(x, t)$ with $t>t^{*}$ along it (generic here means that $\left(m_{\text {in }}, m_{\text {out }}\right)=(1,1)$ [see Proposition 2.5 below for definitions] rather than the non-generic $(1,2)$ points which have lower Hausdorff dimension [4]), all FBW paths through a small neighborhood of $(x, t)$ coalesce quickly with each other.

2. The fact that, as already mentioned, a deterministic space-time point $(x, t)$ is the splice point of the unique FBW path passing through $(x, t)$ is quite natural in the stochastic flow context, as a consequence of Piterbarg's results. Things are contractive going forward in time because of the convergence of forward paths to coalescing Brownian motions. To see that things are expansive at any earlier time, $t_{0}<t$, along the flow line through $(x, t)$, we note that the flow contracts points $\left(x, t_{0}\right)$ by time $t$ towards small regions which in the scaling limit converge to a locally finite set of locations which has zero probability of including the deterministic location $x$. Let $x_{L}$ and $x_{R}$ respectively denote the two locations closest to $x$, respectively to its left and right, toward which points are contracted. Then, in the stochastic flow, in order to find the $\left(x_{0}, t_{0}\right)$ with $t_{0}<t$ which flows exactly into $(x, t)$ one must stay within the small expansive region separating the larger contractive regions which contract towards $x_{L}$ and $x_{R}$.

3. An interesting question raised by our results is whether the fact that every $\gamma$ in the FBW has a unique splice point $\left(x^{*}, t^{*}\right)$ where it changes from expansive behavior for (all) earlier times to contractive behavior for (most) future times has a natural interpretation for stochastic flows (on appropriate space and time scales) that goes beyond the discussion of the previous paragraph.

In the next two sections, we present construction and characterization 
results for the FBW, and then in Section 4, a convergence result, which we apply to the stochastic flows of homeomorphisms of [5, 7] in Section [5] In Section 6, we discuss a maximality property of the forward path of the FBW and its relevance for the characterization of the (standard) Brownian web. Finally, Section 7 contains proofs of two lemmas and a proposition stated in earlier sections.

\section{Construction}

In this section, we construct the FBW, combining backward and forward paths of the double Brownian web (DBW).

\subsection{Preliminaries}

As in [2, 3, 4, we begin with $\left(\overline{\mathbb{R}}^{2}, \rho\right)$, the completion (or compactification) of $\mathbb{R}^{2}$ under the metric $\rho$, where

$$
\rho\left(\left(x_{1}, t_{1}\right),\left(x_{2}, t_{2}\right)\right)=\left|\frac{\tanh \left(x_{1}\right)}{1+\left|t_{1}\right|}-\frac{\tanh \left(x_{2}\right)}{1+\left|t_{2}\right|}\right| \vee\left|\tanh \left(t_{1}\right)-\tanh \left(t_{2}\right)\right| .
$$

$\overline{\mathbb{R}}^{2}$ may be thought as the image of $[-\infty, \infty] \times[-\infty, \infty]$ under the mapping

$$
(x, t) \rightsquigarrow(\Phi(x, t), \Psi(t)) \equiv\left(\frac{\tanh (x)}{1+|t|}, \tanh (t)\right) .
$$

Let $\Pi^{F}$ denote the set of functions $f$ from $[-\infty, \infty]$ to $[-\infty, \infty]$ such that $\Phi(f(t), t)$ is continuous, and let

$$
d^{F}\left(f_{1}, f_{2}\right)=\sup _{-\infty \leq t \leq \infty}\left|\Phi\left(f_{1}(t), t\right)-\Phi\left(f_{2}(t), t\right)\right| .
$$

be a metric on $\Pi^{F}$. Then $\left(\Pi^{F}, d^{F}\right)$ is a complete separable metric space. We will throughout identify an arbitrary element $f$ of $\Pi^{F}$ with its (space-time) path $\gamma=\{(f(s), s):-\infty \leq s \leq \infty\}$.

For the next result (whose proof we leave as an exercise), we use the notation of Section 3 in [4, where forward semipaths $f$ starting at time $t_{0}$ are denoted $\left(f, t_{0}\right)$ and belong to a space $\Pi$ with a similar notation for backward paths $\left(g^{b}, t_{0}\right)$ in $\Pi^{b}$. Further, for $\left[\left(f, t_{0}\right),\left(g^{b}, t_{0}\right)\right] \in \Pi \times \Pi^{b}$ such that $f\left(t_{0}\right)=g^{b}\left(t_{0}\right)$, we let $\left(f, g^{b}, t_{0}\right) \in \Pi^{F}$ denote the path through $\left(f\left(t_{0}\right), t_{0}\right)$ coinciding with $\left(f, t_{0}\right)$ after $t_{0}$, and with $\left(g^{b}, t_{0}\right)$ before $t_{0}$. 
Lemma 2.1 Let $\left\{\left[\left(f_{n}, t_{n}\right),\left(g_{n}^{b}, t_{n}\right)\right] ; n \geq 1\right\}$ be a sequence of paths in $\Pi \times \Pi^{b}$ such that $f_{n}\left(t_{n}\right)=g_{n}^{b}\left(t_{n}\right)$ for $n \geq 1$. If $\left[\left(f_{n}, t_{n}\right),\left(g_{n}^{b}, t_{n}\right)\right] \rightarrow\left[(f, t),\left(g^{b}, t\right)\right]$ as $n \rightarrow \infty$ in $\Pi \times \Pi^{b}$, then $\left(f_{n}, g_{n}^{b}, t_{n}\right) \rightarrow\left(f, g^{b}, t\right)$ as $n \rightarrow \infty$ in $\Pi^{F}$.

Let now $\mathcal{H}^{F}$ denote the set of compact subsets of $\left(\Pi^{F}, d^{F}\right)$, with $d_{\mathcal{H}^{F}}$ the induced Hausdorff metric, i.e.,

$$
d_{\mathcal{H}^{F}}\left(K_{1}, K_{2}\right)=\sup _{g_{1} \in K_{1}} \inf _{g_{2} \in K_{2}} d^{F}\left(g_{1}, g_{2}\right) \vee \sup _{g_{2} \in K_{2}} \inf _{g_{1} \in K_{1}} d^{F}\left(g_{1}, g_{2}\right)
$$

$\left(\mathcal{H}^{F}, d_{\mathcal{H}^{F}}\right)$ is also a complete separable metric space.

The following lemmas will be used in Section 3, they are key to our characterization and hence convergence results. Their proofs are given in Section 7.

Lemma 2.2 Let $\breve{\mathcal{W}} \in \mathcal{H}^{F}$ be noncrossing and such that for a dense countable $\mathcal{D} \subset \mathbb{R}^{2}$ and every $(x, t) \in \mathcal{D}$ there exists a unique path $\gamma_{x, t} \in \mathcal{W}$ passing through $(x, t)$. Then $\breve{\mathcal{W}}$ is determined by the set of semipaths $\mathcal{W}=\left\{W_{x, t}=\right.$ $\left.\left(\gamma_{x, t}(s), s\right)_{s \geq t},(x, t) \in \mathcal{D}\right\}$.

Again we will use the notation of Section 3 of [4]), where $\mathcal{H}$ denotes the Hausdorff space of compact collections of forward semipaths.

Lemma 2.3 For a noncrossing element $\breve{\mathcal{W}} \in \mathcal{H}^{F}$, let $\overrightarrow{\mathcal{W}} \in \mathcal{H}$ be the set of the forward semipaths of $\breve{\mathcal{W}}$, i.e.,

$$
\overrightarrow{\mathcal{W}}=\left\{W_{x, t}=(\gamma(s), s)_{s \geq t}: \gamma \in \breve{\mathcal{W}}, \gamma(t)=x,(x, t) \in \overline{\mathbb{R}}^{2}\right\}
$$

Suppose that for a dense countable $\mathcal{D} \subset \mathbb{R}^{2}$ and every $(x, t) \in \mathcal{D}$ there exists a unique semipath $\gamma_{x, t} \in \overrightarrow{\mathcal{W}}$ starting at $(x, t)$, which furthermore avoids all other points of $\mathcal{D}$. Then $\breve{\mathcal{W}}$ is determined by the set of semipaths $\mathcal{W}=$ $\left\{W_{x, t},(x, t) \in \mathcal{D}\right\}$.

\subsection{Two constructions}

Let $\mathcal{D}$ be an (ordered) dense countable deterministic subset of $\mathbb{R}^{2}$. We give two construction of the FBW, both based on the double Brownian web (DBW). 
1st Construction Let $\mathcal{W}^{D}=\left\{\tilde{W}_{1}, \tilde{W}_{1}^{b}, \tilde{W}_{2}, \tilde{W}_{2}^{b}, \ldots\right\}$ be the DBW skeleton using the set $\mathcal{D}=\left\{\left(x_{1}, t_{1}\right),\left(x_{2}, t_{2}\right), \ldots\right\}$ of space-time points as starting points for these coalescing/reflecting forward/backward Brownian motions (see the beginning of Section 3 of [4). Define the FBW skeleton $\mathcal{W}^{F}=\left\{\tilde{W}_{1}^{F}, \tilde{W}_{2}^{F}, \ldots\right\}$ by

$$
\tilde{W}_{j}^{F}= \begin{cases}\tilde{W}_{j}^{b}(t), & t \leq t_{j} \\ \tilde{W}_{j}(t), & t \geq t_{j} .\end{cases}
$$

Finally, let $\overline{\mathcal{W}}^{F}$ be the closure of $\mathcal{W}^{F}$ in $\left(\Pi^{F}, d^{F}\right)$.

Proposition 2.4 $\overline{\mathcal{W}}^{F}$ is compact (and so is an $\left(\mathcal{H}^{F}, d_{\mathcal{H}^{F}}\right)$-valued random variable) and its distribution does not depend on $\mathcal{D}$ or its ordering.

2nd Construction As in 4, we denote by $\overline{\mathcal{W}}$ and $\overline{\mathcal{W}}^{b}$ the forward and backward Brownian webs, which are compact subsets of semipaths of their respective spaces $\Pi$ and $\Pi^{b}$.

Proposition 2.5 $\overline{\mathcal{W}}^{F}$ consists of the two "trivial" paths that are identically $+\infty$ or $-\infty$ for all $t$, plus the following collection of paths constructed from the $D B W \overline{\mathcal{W}}^{D}=\left(\overline{\mathcal{W}}, \overline{\mathcal{W}}^{b}\right)$. For each $\left(x^{*}, t^{*}\right) \in \mathbb{R}^{2}$, of type $\left(m_{\text {in }}, m_{\text {out }}\right)^{1}$, let $W_{1}, \ldots, W_{m_{\text {out }}}$ be the forward paths from $\left(x^{*}, t^{*}\right)$, and $W_{1}^{b}, \ldots, W_{m_{\text {in }}+1}^{b}$ be the backward paths from $\left(x^{*}, t^{*}\right)$. Then take every path of the form

$$
W_{i j}^{F}=\left(W_{i}, W_{j}^{b}, t^{*}\right)= \begin{cases}W_{i}(t), & t \geq t^{*} \\ W_{j}^{b}(t), & t \leq t^{*}\end{cases}
$$

except, when $\left(m_{\text {in }}, m_{\text {out }}\right)=(1,2)$ (see Figure 11), do not take the unique choice of $i, j$ for which $W_{i j}^{F}$ would cross the forward path passing through $\left(x^{*}, t^{*}\right)$ [i.e., in Figure 1, do not take the $W_{i j}^{F}$ from Southwest to Northeast].

Remark 2.6 The trivial paths, namely the identically $-\infty$ one and the identically $+\infty$ one, are also splicings of paths in the DBW. Indeed, for any $t^{*}$, the identically $+\infty$ path is the splicing of the forward path (in $\overline{\mathcal{W}}$ ) starting at

\footnotetext{
${ }^{1} m_{\text {in }}$ denotes the number of forward paths of $\overline{\mathcal{W}}^{D}$ starting before $t^{*}$, not coalescing up to right before $t^{*}$, and passing through $\left(x^{*}, t^{*}\right)$, and $m_{\text {out }}$ denotes the number of forward paths of $\overline{\mathcal{W}}^{D}$ starting at $\left(x^{*}, t^{*}\right)$; see, e.g., Section 3 of 4 .
} 




Figure 1: A schematic diagram of a point $\left(x^{*}, t^{*}\right)$ of type $\left(m_{\text {in }}, m_{\text {out }}\right)=(1,2)$.

$\left(+\infty, t^{*}\right)$ with the backward path $\left(\right.$ in $\left.\overline{\mathcal{W}}^{b}\right)$ from $\left(+\infty, t^{*}\right)$. One difference between the trivial and the nontrivial paths is that the latter have a well-defined unique splice point (in $\mathbb{R}^{2}$ ), while for the latter all their points can be seen as splice points.

Remark 2.7 Every path $\gamma$ in the FBW consists of a backward path from the $D B W$ between times $t=-\infty$ and $t=$ some $t^{*}(\gamma)$, spliced with a forward path from $t^{*}(\gamma)$ to $t=+\infty$. Every $\left(x^{*}, t^{*}\right)$ in $\mathbb{R}^{2}$ is a splice point of of one or more $\gamma^{\prime}$ 's from the $F B W$; the number of $\gamma^{\prime}$ 's for a given splice point $\left(x^{*}, t^{*}\right)$, determined by the type $\left(m_{\mathrm{in}}^{*}, m_{\mathrm{out}}^{*}\right)$ of $\left(x^{*}, t^{*}\right)$, is $\left(m_{\mathrm{in}}^{*}+1\right) m_{\mathrm{out}}^{*}$, except that for a point of type $(1,2)$, that number is only 3 , rather than 4 .

As one follows a backward path $\gamma^{b}$ forward in time to some $\left(x^{\#}, t^{\#}\right)$, the number of choices of how to continue it (locally) as a path in the FBW depends on the type $\left(m_{\mathrm{in}}^{\#}, m_{\text {out }}^{\#}\right)$ of $\left(x^{\#}, t^{\#}\right)$, as well as on whether $\gamma^{b}$ is part of a backward path passing through $\left(x^{\#}, t^{\#}\right)$ [rather than originating at $\left(x^{\#}, t^{\#}\right)$ ]. If $\gamma^{b}$ is not part of a backward path passing through $\left(x^{\#}, t^{\#}\right)$ (this is the case if $m_{\text {out }}^{\#}=1$, or sometimes when $\left(m_{\text {in }}^{\#}, m_{\text {out }}^{\#}\right)=(1,2)-$ e.g. if $\gamma^{b}$ is the Southwest path in Figure 1, then $\left(x^{\#}, t^{\#}\right)$ must be a splice point and there is a unique choice of forward path to splice into. If $\gamma^{b}$ is rather part of a backward 
path passing through $\left(x^{\#}, t^{\#}\right)$, then $\left(x^{\#}, t^{\#}\right)$ can be a splice point with $m_{\text {out }}^{\#}$ choices of forward path to splice into, or $\gamma^{b}$ can be continued to any of $m_{\mathrm{out}}^{\#}-1$ possible backward path continuations. E.g., for $\left(m_{\text {in }}^{\#}, m_{\text {out }}^{\#}\right)=(0,3)$, there are 5 possible continuations, with 3 of them corresponding to splice points. Once a splice point has been chosen, and a continuation to a forward path has been made, that forward path is followed until $t=+\infty$ with no further choices.

Note that if a path $\gamma$ in the FBW touches a point $\left(\gamma\left(t_{0}\right), t_{0}\right)$ with $m_{\text {out }}=$ 1 , then for $t \geq t_{0}, \gamma(t)$ follows the unique forward path in the $B W$ from $\left(\gamma\left(t_{0}\right), t_{0}\right)$.

Proof of Proposition 2.5 Let $\hat{\mathcal{W}}$ be the set of paths described in the statement. Also let $\overline{\mathcal{W}}^{F}$ denote the closed set of paths in $\left(\Pi^{F}, d^{F}\right)$ from our first construction for some particular $\mathcal{D}$; as we have not yet proved Proposition 2.4 $\overline{\mathcal{W}}^{F}$ could a priori depend on $\mathcal{D}$. We show $(i) \overline{\mathcal{W}}^{F} \subset \hat{\mathcal{W}}$ and $(i i) \hat{\mathcal{W}} \subset \overline{\mathcal{W}}^{F}$.

(i) Take a path $W^{F} \in \overline{\mathcal{W}}^{F}$ and a sequence $\tilde{W}_{j_{1}}^{F}, \tilde{W}_{j_{2}}^{F}, \ldots$ of paths in $\mathcal{W}^{F}$ converging to $W^{F}$. By the compactness of $\overline{\mathbb{R}}^{2}$, the sequence of splice points $\left(x_{j_{1}}, t_{j_{1}}\right),\left(x_{j_{2}}, t_{j_{2}}\right), \ldots$ of $\tilde{W}_{j_{1}}^{F}, \tilde{W}_{j_{2}}^{F}, \ldots$ has a convergent subsequence $\left(x_{j_{1}^{\prime}}, t_{j_{1}^{\prime}}\right),\left(x_{j_{2}^{\prime}}, t_{j_{2}^{\prime}}\right), \ldots$ with a limit point $\left(x^{*}, t^{*}\right) \in \overline{\mathbb{R}}^{2}$. It readily follows that $\left(\tilde{W}_{j_{i}^{\prime}}\right)_{i \geq 1}$ converges in $\Pi$ to a forward path $W$ starting in $\left(x^{*}, t^{*}\right)$, that $\left(\tilde{W}_{j_{i}^{\prime}}^{b}\right)_{i \geq 1}$ converges in $\Pi^{b}$ to a backward path $W^{b}$ starting in $\left(x^{*}, t^{*}\right)$, and that $W^{F}$ is the splicing of $W$ and $W^{b}$. By the compactness of the double Brownian web, $W \in \overline{\mathcal{W}}$ and $W^{b} \in \overline{\mathcal{W}}^{b}$. It is also easy to see that $\left(W, W^{b}, t^{*}\right)$ cannot be the one case of splicing ruled out in the definition of $\hat{\mathcal{W}}$. Thus, $W^{F} \in \hat{\mathcal{W}}$. (We note that $W^{F}$ is a trivial path iff $\left(x^{*}, t^{*}\right) \in \overline{\mathbb{R}}^{2} \backslash \mathbb{R}^{2}$.)

(ii) Take a path $\hat{W}^{F} \in \hat{\mathcal{W}}$, and let $\left(x^{*}, t^{*}\right)$ be its splice point (taken to be $( \pm \infty, 0)$ for the identically $\pm \infty$ trivial paths, respectively), so that $\hat{W}^{F}=\left(\hat{W}, \hat{W}^{b}, t^{*}\right)$.

If $\left(x^{*}, t^{*}\right)$ is not of type $(1,2)^{2}$, then either $\hat{W}$ is the unique path in $\overline{\mathcal{W}}$ from $\left(x^{*}, t^{*}\right)$, or $\hat{W}^{b}$ is the unique path in $\overline{\mathcal{W}}^{b}$ starting from $\left(x^{*}, t^{*}\right)$. Suppose we are in the the latter case (the former case can be handled similarly). Then, since $\overline{\mathcal{W}}$ is the closure of the set of its paths starting from points in $\mathcal{D}$, there exists a sequence $\left(x_{j_{1}}, t_{j_{1}}\right),\left(x_{j_{2}}, t_{j_{2}}\right), \ldots$ in $\mathcal{D}$ approaching $\left(x^{*}, t^{*}\right)$ such that $\left(\tilde{W}_{j_{i}}\right)_{i \geq 1}$ converges in $\Pi$ to $\hat{W}$. Now by the compactness of $\overline{\mathcal{W}}^{b}$ and the uniqueness property of $\hat{W}^{b}$ just mentioned, $\left(\tilde{W}_{j_{i}}^{b}\right)_{i \geq 1}$ converges in $\Pi^{b}$ to $\hat{W}^{b}$. By Lemma 2.1, $\left(\tilde{W}_{j_{i}}^{F}\right)_{i \geq 1}$ converges in $\Pi^{F}$ to $\hat{W}^{F}$, and thus $\hat{W}^{F} \in \overline{\mathcal{W}}^{F}$.

\footnotetext{
${ }^{2}$ This happens in particular in the case of $( \pm \infty, 0)$, which is of type $(1,1)$.
} 
Suppose now that $\left(x^{*}, t^{*}\right)$ is a left-handed type $(1,2)$ point as in Figure 1 (see Remark 3.11 in [4] the right-handed case is treated similarly), and let $W_{1}, W_{2}$ be the two paths in $\overline{\mathcal{W}}$ starting from $\left(x^{*}, t^{*}\right)$, and $W_{1}^{b}, W_{2}^{b}$ be the two paths in $\overline{\mathcal{W}}^{b}$ starting from $\left(x^{*}, t^{*}\right)$, with $W_{1}$ to the left of $W_{2}$, and $W_{1}^{b}$ to the left of $W_{2}^{b}$. Let also $\check{W}_{1}$ be any path in $\overline{\mathcal{W}}$ starting before $t^{*}$ and passing through $\left(x^{*}, t^{*}\right)$, and $\check{W}_{2}^{b}$ be any path in $\overline{\mathcal{W}}^{b}$ from above $t^{*}$ passing through $\left(x^{*}, t^{*}\right)$. ( $\check{W}_{1}$ and $\check{W}_{2}^{b}$ coincide with $W_{1}$ and $W_{2}^{b}$ above and below $t^{*}$ respectively.) We then have that $\left(\hat{W}, \hat{W}^{b}, t^{*}\right)=\left(W_{i}, W_{j}^{b}, t^{*}\right)$, for some $i, j=1,2$, but $(i, j) \neq(2,1)$.

If $(i, j)=(1,1)$ and we take a sequence $\left(x_{j_{1}}, t_{j_{1}}\right),\left(x_{j_{2}}, t_{j_{2}}\right), \ldots$ in $\mathcal{D}$ approaching $\left(x^{*}, t^{*}\right)$ such that $\left(x_{j_{i}}, t_{j_{i}}\right)$ is to the left of $\check{W}_{1}$ for all $i$, then $\tilde{W}_{j_{i}} \rightarrow W_{1}$, and $\tilde{W}_{j_{i}}^{b} \rightarrow W_{1}^{b}$ as $i \rightarrow \infty$.

If $(i, j)=(2,2)$ and we take a sequence $\left(x_{j_{1}^{\prime}}, t_{j_{1}^{\prime}}\right),\left(x_{j_{2}^{\prime}}, t_{j_{2}^{\prime}}\right), \ldots$ in $\mathcal{D}$ approaching $\left(x^{*}, t^{*}\right)$ such that $\left(x_{j_{i}^{\prime}}, t_{j_{i}^{\prime}}\right)$ is to the right of $\check{W}_{2}^{b}$ for all $i$, then $\tilde{W}_{j_{i}^{\prime}} \rightarrow W_{2}$, and $\tilde{W}_{j_{i}^{\prime}}^{b} \rightarrow W_{2}^{b}$ as $i \rightarrow \infty$.

If $(i, j)=(1,2)$, then if we take a sequence $\left(x_{j_{1}^{\prime \prime}}, t_{j_{1}^{\prime \prime}}\right),\left(x_{j_{2}^{\prime \prime}}, t_{j_{2}^{\prime \prime}}\right), \ldots$ in $\mathcal{D}$ approaching $\left(x^{*}, t^{*}\right)$ such that $\left(x_{j_{i}^{\prime \prime}}, t_{j_{i}^{\prime \prime}}\right)$ is between $\check{W}_{1}$ and $\check{W}_{2}^{b}$ for all $i$, then $\tilde{W}_{j_{i}^{\prime \prime}} \rightarrow W_{1}$, and $\tilde{W}_{j_{i}^{\prime \prime}}^{b} \rightarrow W_{2}^{b}$ as $i \rightarrow \infty$.

In all cases, we conclude from Lemma 2.1 that $\hat{W}^{F}$ is the limit of a sequence in $\overline{\mathcal{W}}^{F}$.

Proof of Proposition 2.4 Let $W_{n}^{F}=\left(W_{n}, W_{n}^{b}, t_{n}\right), n \geq 1$, be a sequence of paths in $\overline{\mathcal{W}}^{F}$ with splice points $\left(x_{n}, t_{n}\right), n \geq 1$, respectively. By the compactness of $\overline{\mathbb{R}}^{2},\left(x_{n^{\prime}}, t_{n^{\prime}}\right) \rightarrow(x, t) \in \overline{\mathbb{R}}^{2}$ as $n^{\prime} \rightarrow \infty$ for a subsequence $\left(x_{n^{\prime}}, t_{n^{\prime}}\right)$ of $\left(x_{n}, t_{n}\right)$. By the compactness of $\overline{\mathcal{W}}^{D},\left(W_{n^{\prime \prime}}, W_{n^{\prime \prime}}^{b}\right) \rightarrow\left(W, W^{b}\right)$ as $n^{\prime \prime} \rightarrow \infty$ for a a subsequence $\left(x_{n^{\prime \prime}}, t_{n^{\prime \prime}}\right)$ of $\left(x_{n^{\prime}}, t_{n^{\prime}}\right)$, with $\left(W, W^{b}\right) \in \overline{\mathcal{W}}^{D}$. Since clearly $W(t)=W^{b}(t)=x$, we have by Lemma 2.1 that $W_{n^{\prime \prime}}^{F} \rightarrow\left(W, W^{b}, t\right) \in \overline{\mathcal{W}}^{F}$ as $n^{\prime \prime} \rightarrow \infty$. The nondependence of the distribution of $\overline{\mathcal{W}}^{F}$ on $\mathcal{D}$ is immediate from the same property of $\overline{\mathcal{W}}^{D}$ and Proposition 2.5.

\section{Characterization}

In this section we state some results characterizing the distribution of the FBW. We will use the following fact about the double Brownian web, whose proof is found in Section 7. 
Proposition 3.1 Let $\overline{\mathcal{W}} \in\left(\mathcal{H}, \mathcal{F}_{\mathcal{H}}\right)$ be a standard Brownian web. Then there exists a standard double Brownian web $\hat{\mathcal{W}}^{D}=\left(\hat{\mathcal{W}}, \hat{\mathcal{W}}^{b}\right) \in\left(\mathcal{H}^{D}, \mathcal{F}_{\mathcal{H}^{D}}\right)$ such that a.s. $\hat{\mathcal{W}}=\overline{\mathcal{W}}$.

Theorem 3.2 There is an $\left(\mathcal{H}^{F}, d_{\mathcal{H}^{F}}\right)$-valued random variable $\overline{\mathcal{W}}^{F}$ whose distribution is uniquely determined by the following properties.

(a) Almost surely the paths of $\overline{\mathcal{W}}^{F}$ are noncrossing (although they may touch, including coalescing and bifurcating).

$\left(b_{1}\right)$ From any deterministic point $(x, t) \in \mathbb{R}^{2}$, there is almost surely a unique path $W_{x, t}^{F}$ passing through $x$ at time $t$.

$\left(b_{2}\right)$ For any deterministic $n,\left\{\left(x_{1}, t_{1}\right), \ldots,\left(x_{n}, t_{n}\right)\right\}$, the joint distribution of the semipaths $\left\{W_{x_{j}, t_{j}}^{F}(t), t \geq t_{j}, j=1, \ldots, n\right\}$ is that of coalescing Brownian motions (with unit diffusion constant).

Remark $3.3\left(b_{1}\right)$ and $\left(b_{2}\right)$ may together be replaced by the following weaker condition.

(b) For a given deterministic countable dense set $\mathcal{D} \in \mathbb{R}^{2}$, there exist (not necessarily unique a priori) paths $W_{x_{j}, t_{j}}^{F}$ in $\overline{\mathcal{W}}^{F}$ for each $\left(x_{j}, t_{j}\right)$ in $\mathcal{D}$ such that $\left\{W_{x_{j}, t_{j}}^{F}(t), t \geq t_{j}, j=1,2, \ldots\right\}$ is distributed as coalescing Brownian motions (with unit diffusion constant) starting at the points of $\mathcal{D}$.

Proof of Theorem 3.2 Condition $\left(b_{2}\right)$ implies that for any fixed deterministic countable dense $\mathcal{D}$, the set of semipaths $\mathcal{W}=\left\{\left(W_{x, t}^{F}(s)\right)_{s \geq t},(x, t) \in \mathcal{D}\right\}$ is distributed as a Brownian web skeleton. Thus $\overline{\mathcal{W}}$, the closure of $\mathcal{W}$, is a standard Brownian web. By Proposition 3.1 there exists a dual Brownian web $\overline{\mathcal{W}}^{b}$ such that $\left(\overline{\mathcal{W}}, \overline{\mathcal{W}}^{b}\right)$ is a double Brownian web. Condition $\left(b_{1}\right)$ implies that there is a unique backward semipath of $\overline{\mathcal{W}}^{F}$ starting from each point of $\mathcal{D}$. Thence Lemma 2.2 and Proposition 2.5 (or Proposition 2.4) yield the result.

Proof of Remark 3.3 Condition (b) implies that the set of semipaths $\mathcal{W}=\left\{\left(W_{x, t}^{F}(s)\right)_{s \geq t},(x, t) \in \mathcal{D}\right\}$ is a Brownian web skeleton (the a.s. uniqueness of the semipath starting in each point of $\mathcal{D}$ follows by the trapping argument used in the proof of Proposition 3.1 of [3] (arXiv version)). The proof continues like the one of Theorem 3.2 above, using Lemma 2.3 instead of Lemma 2.2. 


\section{Convergence}

In this section we establish convergence criteria for a sequence of $\left(\mathcal{H}^{F}, d_{\mathcal{H}^{F}}\right)$ valued random variables to converge to the FBW. These criteria are then applied in the next section to show that the diffusively rescaled stochastic flow of homeomorphisms of [5, 7] converges to the FBW as the scale parameter goes to 0 .

Theorem 4.1 Let $\mathcal{X}_{1}, \mathcal{X}_{2}, \ldots$ be a sequence of $\left(\mathcal{H}^{F}, d_{\mathcal{H}^{F}}\right)$-valued random variables such that

$(\bar{a})$ almost surely, the paths of each $\mathcal{X}_{m}$ are noncrossing, and

(b) for a given deterministic countable dense set $\mathcal{D} \in \mathbb{R}^{2}$, there exists for each $\left(x_{j}, t_{j}\right) \in \mathcal{D}$ a path $\theta_{m}^{x_{j}, t_{j}}$ in $\mathcal{X}_{m}$ such that the collection of semipaths $\mathcal{V}_{m}:=\left\{\left(\theta_{m}^{x_{j}, t_{j}}(t), t \geq t_{j}\right),\left(x_{j}, t_{j}\right) \in \mathcal{D}\right\}$ converges in distribution as $m \rightarrow \infty$ to coalescing Brownian motions (with unit diffusion constant) starting in $\mathcal{D}$.

Then $\mathcal{X}_{m}$ converges in distribution as $m \rightarrow \infty$ to the $F B W$.

Proof We claim that $\left(\mathcal{X}_{m}, m \geq 1\right)$ is tight. Then, conditions $(\bar{a})-(\bar{b})$ and Remark 3.3 imply that all limit points of $\left(\mathcal{X}_{m}, m \geq 1\right)$ are distributed as the FBW.

To justify the tightness claim, let $\mathcal{V}_{m}$ (resp., $\mathcal{V}_{m}^{b}$ ) denote the collection of all forward (resp., backward) semipaths of $\mathcal{X}_{m}$. By Proposition B.3 of [3], we have tightness of $\mathcal{V}_{m}$ in $\left(\mathcal{H}, d_{\mathcal{H}}\right)$ and it then suffices to verify that $\left(\mathcal{V}_{m}^{b}, m \geq 1\right)$ is tight in $\left(\mathcal{H}^{b}, d_{\mathcal{H}^{b}}\right)$. This readily implies tightness of $\left\{\left(\mathcal{V}_{m}, \mathcal{V}_{m}^{b}\right), m \geq 1\right\}$ in $\left(\mathcal{H}^{D}, d_{\mathcal{H}^{D}}\right)$, and this and Lemma 2.1 imply tightness of $\left(\mathcal{X}_{m}, m \geq 1\right)$.

Now tightness of $\left(\mathcal{V}_{m}^{b}, m \geq 1\right)$ follows by the very same blocking argument used in the proof of Proposition 3.1 given in Section 7 below to verify the tightness condition $\left(T_{1}\right)$ from Appendix B of [3].

\section{Stochastic flows and the FBW}

Let $\Xi=\left\{\xi_{s t}, s \leq t\right\}$ be an isotropic stochastic flow of homeomorphisms with covariance structure $B$. This means, among other things, that for every $s \in \mathbb{R}$

$$
\left(\xi_{s t}\left(x_{1}\right), \ldots, \xi_{s t}\left(x_{n}\right) ; t \geq s\right)
$$


is an $R^{n}$-diffusion starting at $\left(x_{1}, \ldots, x_{n}\right)$ with generator

$$
\frac{B(0)}{2} \sum^{n} \frac{\partial^{2}}{\partial y_{i}^{2}}+\sum_{i<j}^{n} B\left(y_{i}-y_{j}\right) \frac{\partial^{2}}{\partial y_{i} \partial y_{j}}
$$

Piterbarg [6, 7] studied expansions/contractions of $\Xi$ via the $\delta \rightarrow 0$ limit of

$$
\xi_{t}^{\delta}(x) \equiv \delta \xi_{0 t \delta^{-2}}\left(x \delta^{-1}\right) .
$$

It follows from his results that when $B$ is nice (with $B(x) \rightarrow 0$ as $|x| \rightarrow \infty$; see conditions (B1-B4) in [7]), then for all $\left(x_{1}, t_{1}\right), \ldots,\left(x_{n}, t_{n}\right) \in \mathbb{R}^{2}$

$$
\left\{\left(\xi^{\delta}\left(x_{1}, t_{1}\right), s \geq t_{1}\right), \ldots,\left(\xi^{\delta}\left(x_{n}, t_{n}\right), s \geq t_{n}\right)\right\}
$$

converges weakly as $\delta \rightarrow 0$ to coalescing Brownian motions starting from $\left(x_{1}, t_{1}\right), \ldots,\left(x_{n}, t_{n}\right) \in \mathbb{R}^{2}$, where for $i=1, \ldots, n$ and $\delta>0$,

$$
\xi^{\delta}\left(x_{1}, t_{1}\right)=\xi_{t_{i} \delta^{-2}, s \delta^{-2}}\left(x_{i} \delta^{-1}\right)
$$

(see Theorem 6 in [7]; there the case $t_{i}=$ constant is stated, from which the general case readily follows).

Since these flows are noncrossing, the above and Theorem 4.1 immediately imply the following result.

Theorem 5.1 For $\delta>0$, let $\mathcal{X}_{\delta}=\left\{\left[\delta \xi_{0 t \delta^{-2}}\left(x \delta^{-1}\right), t \in \mathbb{R}\right], x \in \mathbb{R}\right\}$ as in [7]. Then $\mathcal{X}_{\delta}$ converges in distribution to the $F B W$ as $\delta \rightarrow 0$.

\section{The forward full Brownian web}

In this section, we use the FBW, or rather its forward part, to provide an example clarifying the significance of a condition in characterization results for the (standard) Brownian web (see, e.g., Condition (ii) in Theorem 2.1 or Condition $\left(i i^{\prime}\right)$ in Theorem 4.1 [Theorem 4.5 in the arXiv version] of [3] and the remark following Theorem 2.1 there; see also Remark 6.2 below).

For a path $\gamma \in \mathcal{W}^{F}$ and $t \in[-\infty, \infty]$, let $\gamma_{t}$ denote the forward semipath starting at $(\gamma(t), t)$, and consider the path collection

$$
\mathcal{W}^{F F}:=\left\{\gamma_{t}: \gamma \in \mathcal{W}^{F}, t \in[-\infty, \infty]\right\}
$$


The next theorem says that $\mathcal{W}^{F F}$ is, in a natural sense, the maximal collection of (noncrossing) forward paths containing the standard Brownian web; thus it is the full forward as well as the forward full Brownian web. Indeed, $\mathcal{W}^{F F}$ is the maximal collection satisfying properties (0)-(iii) below while the standard $\mathrm{BW}$ is the minimal such collection. In particular, for $-\infty<s<t<\infty$, the set of $x$ such that there is a (forward) semipath passing through both $\mathbb{R} \times\{s\}$ and $(x, t)$ is locally finite for the BW while it consists of all of $\mathbb{R}$ for $\mathcal{W}^{F F}$.

Theorem 6.1 $\mathcal{W}^{F F}$ has the following properties.

(o) $\mathcal{W}^{F F}$ is a.s. compact in $(\Pi, d)$, the space of forward semipaths ${ }^{3}$;

(i) the paths of $\mathcal{W}^{F F}$ are noncrossing;

(ii) from any deterministic point $(x, t)$ in $\mathbb{R}^{2}$, there is almost surely a unique path $W_{x, t}$ in $\mathcal{W}^{F F}$ starting from $(x, t)$;

(iii) for any deterministic $n,\left(x_{1}, t_{1}\right), \ldots,\left(x_{n}, t_{n}\right)$, the joint distribution of $W_{x_{1}, t_{1}}, \ldots, W_{x_{n}, t_{n}}$ is that of coalescing Brownian motions (with unit diffusion constant);

(iv) $\mathcal{W}^{F F}$ strictly contains a version of the standard Brownian web $\overline{\mathcal{W}}$;

(v) if $\hat{\mathcal{W}}$ satisfies properties (o)-(iii) above, then there exists a version $\mathcal{W}^{*}$ of $\mathcal{W}^{F F}$ such that $\hat{\mathcal{W}} \subset \mathcal{W}^{*}$.

Remark 6.2 Theorem 2.1 of [3] establishes that under conditions (ii) and (iii) of Theorem [6.1 above, a $\left(\mathcal{H}, d_{\mathcal{H}}\right)$-random variable is a (standard) Brownian web provided that a further condition (alluded to at the beginning of this section) is satisfied, namely that it is almost surely the closure of the set of its paths starting at a deterministic countable subset of $\overline{\mathbb{R}}^{2}$. Theorem [6.1 above makes clear that without the latter condition, we can have $\left(\mathcal{H}, d_{\mathcal{H}}\right)$ random variables satisfying conditions (ii) and (iii) of Theorem [6.1 other than the Brownian web (as established in Theorem 3.1 [Theorem 3.6 in the arXiv version] of [3], these $\left(\mathcal{H}, d_{\mathcal{H}}\right)$-random variables must be stochastically bigger than the Brownian web; that is of course the case with $\mathcal{W}^{F F}$, as stated in (iv) above).

\footnotetext{
${ }^{3}$ See Section 3 of $[3$ for the precise definition of the metric $d$ for semipaths.
} 
Proof Properties (o)-(iii) have already been discussed in/follow readily from our construction (e.g., the 2nd one).

(iv) That $\mathcal{W}^{F F} \supset \mathcal{W}$ is immediate from our 2 nd construction. That $\mathcal{W}^{F F}$ has strictly more paths than $\overline{\mathcal{W}}$ is seen, e.g., in that there are infinitely many paths of $\mathcal{W}^{F F}$ starting at any $(0,2)$-point of $\overline{\mathcal{W}}$, namely, apart from the two paths $W_{1}$ and $W_{2}$, say, of $\overline{\mathcal{W}}$ starting at such a point, all forward semipaths of $\mathcal{W}^{F}$ obtained by splicing paths of $\overline{\mathcal{W}}$ starting at points above the $(0,2)$-point and between $W_{1}$ and $W_{2}$, with the path segments in the dual to $\overline{\mathcal{W}}$ starting at those points and going down to the $(0,2)$-point.

(v) For a deterministic dense countable $\mathcal{D} \subset \mathbb{R}^{2}$, let $\mathcal{W}$ be the set of paths of $\hat{\mathcal{W}}$ starting from $\mathcal{D}$. Then, by (ii)-(iii), $\overline{\mathcal{W}}$, the closure of $\mathcal{W}$, is a Brownian web. By Proposition 3.1 there exists a dual Brownian web $\overline{\mathcal{W}}^{b}$ such that $\overline{\mathcal{W}}^{D}=\left(\overline{\mathcal{W}}, \overline{\mathcal{W}}^{b}\right)$ is a DBW. Let now $\overline{\mathcal{W}}^{F}$ be the FBW obtained from $\overline{\mathcal{W}}^{D}$ (as in our 2nd construction), and $\overline{\mathcal{W}}^{F F}$ be the forward full Brownian web obtained from $\overline{\mathcal{W}}^{F}$ (as in (6.1) above). We claim that $\hat{\mathcal{W}} \subset \overline{\mathcal{W}}^{F F}$. To justify that, we will extend $\hat{\mathcal{W}}$ to a subset of $\mathcal{H}^{F}$ and then use (o)-(i) and Lemma 2.2.

To make that extension, for each $(x, t) \in \mathbb{R}^{2}$ we choose a single backward path $\hat{W}_{x, t}^{b}$ in $\overline{\mathcal{W}}^{b}$ from $(x, t)$, with the proviso that if $(x, t)$ is a $(1,2)$-point of $\overline{\mathcal{W}}^{D}$, then this single backward path must be chosen in the unique way so that the splicings of $\hat{W}_{x, t}^{b}$ with the forward paths of $\overline{\mathcal{W}}$ from $(x, t)$ do not cross any of the paths of $\mathcal{W}^{D}$ passing through $(x, t)$ (e.g., for the $(1,2)$-point of Figure 1, we choose the Southwest backward path). For each path $\hat{W} \in \hat{\mathcal{W}}$ starting at $(x, t)$, let $\gamma=\gamma(\hat{W})$ be the path in $\Pi^{F}$ obtained as the splicing of $\hat{W}$ and $\hat{W}_{x, t}^{b}$, and consider the sets $\mathcal{W}^{*}=\{\gamma(\hat{W}): \hat{W} \in \hat{\mathcal{W}}\}$, and its closure $\overline{\mathcal{W}}^{*}$. We claim that $\mathcal{W}^{*}$ is noncrossing, since $\hat{\mathcal{W}}$ is noncrossing by (i), and if $\hat{W} \in \hat{\mathcal{W}}$ crossed any $\hat{W}_{x, t}^{b} \in \overline{\mathcal{W}}^{b}$, then there would have to be a path in $\mathcal{W}$ crossing $\hat{W}_{x, t}^{b}$, which cannot happen since $\mathcal{W}$ is part of the DBW $\overline{\mathcal{W}}^{D}$; we have also to rule out that $\hat{W} \in \hat{\mathcal{W}}$ crosses a path $\gamma \in \mathcal{W}^{*}$ at a splicing point $(x, t)$ of $\gamma$, but that would mean that $(x, t)$ is a $(1,2)$-point of $\overline{\mathcal{W}}^{D}$ and that the choice of $\hat{W}_{x, t}^{b}$ did not obey the above proviso. Compactness of $\overline{\mathcal{W}}^{*}$ follows readily from (o) and the precompactness of $\left\{\hat{W}_{x, t}^{b},(x, t) \in \overline{\mathbb{R}}^{2}\right\}$. The claim of $(v)$ readily follows. 


\section{$7 \quad$ Several proofs}

We begin with a Lemma that will be used in the proof of Lemma 2.2 ,

Lemma 7.1 Under the hypotheses of Lemma 2.2, $\breve{\mathcal{W}}$ is the closure of $\left\{\gamma_{x, t}\right.$ : $(x, t) \in \mathcal{D}\}$.

Proof Let $\Gamma$ denote $\left\{\gamma_{x, t}:(x, t) \in \mathcal{D}\right\}$ and take any path $\gamma \in \breve{\mathcal{W}}$. The lemma follows if we can show that $\gamma$ is in the closure of $\Gamma$. Let now $\left\{q_{i}, i=1,2, \ldots\right\}$ be an enumeration of the rational numbers of $\mathbb{R}$. We start by taking a sequence $\left(s_{i}^{(1)}\right)_{i=1}^{\infty}$ such that for $i=1,2, \ldots$ we have $s_{i}^{(1)}=\left(x_{i}^{(1)}, t_{i}^{(1)}\right) \in \mathcal{D}$, $x_{i}^{(1)} \geq \gamma\left(t_{i}^{(1)}\right)$ and $s_{i}^{(1)} \rightarrow\left(\gamma\left(q_{1}\right), q_{1}\right)$ as $i \rightarrow \infty$. Let $\gamma^{(1)}$ be a limit (in $\Pi^{F}$ ) of $\gamma_{x_{i}^{(1)}, t_{i}^{(1)}}$. It is clear that $\gamma^{(1)}$ is in $\breve{\mathcal{W}}$, that $\gamma^{(1)}(s) \geq \gamma(s) \forall s \in \mathbb{R}$, and that $\gamma^{(1)}\left(q_{1}\right)=\gamma\left(q_{1}\right)$.

Let us suppose that for $n \geq 1$, we have chosen paths $\gamma^{(j)}, j=1, \ldots, n$, such that

$$
\gamma^{(j)} \in \breve{\mathcal{W}}, \gamma^{(j)}(s) \geq \gamma(s) \forall s \in \mathbb{R}, \text { and } \gamma^{(j)}\left(q_{i}\right)=\gamma\left(q_{i}\right) \text { for } 1 \leq i \leq j \leq n
$$

we now obtain a path $\gamma^{(n+1)}$ such (7.1) holds with $n$ replaced by $n+1$. If $\gamma^{(n)}\left(q_{n+1}\right)=\gamma\left(q_{n+1}\right)$, then let $\gamma^{(n+1)}=\gamma^{(n)}$; otherwise, we must have $\gamma^{(n)}\left(q_{n+1}\right)>\gamma\left(q_{n+1}\right)$. We can then choose a sequence $s_{i}^{(n+1)}=\left(x_{i}^{(n+1)}, t_{i}^{(n+1)}\right)$ in $\mathcal{D}$ such that $\gamma\left(t_{i}^{(n+1)}\right) \leq x_{i}^{(n+1)}<\gamma^{(n)}\left(t_{i}^{(n+1)}\right)$ and $s_{i}^{(n+1)} \rightarrow\left(\gamma\left(q_{n+1}\right), q_{n+1}\right)$ as $i \rightarrow \infty$. Now let $\gamma^{(n+1)}$ be a limit (in $\Pi^{F}$ ) of $\gamma_{x_{i}^{(n+1)}, t_{i}^{(n+1)} \text {. }}$

Finally, let $\bar{\gamma}$ be a limit (in $\Pi^{F}$ ) of $\left\{\gamma^{(n)}: n \geq 1\right\}$. Then we must have $\bar{\gamma}(s) \geq \gamma(s) \forall s \in \mathbb{R}$ while $\bar{\gamma}\left(q_{i}\right)=\gamma\left(q_{i}\right)$ for $i=1,2, \ldots$, and thus $\bar{\gamma}=\gamma$.

Proof of Lemma 2.2 Write $\mathcal{D}=\left\{\left(x_{i}, t_{i}\right), i=1,2, \ldots\right\}$ and $W_{i}=W_{x_{i}, t_{i}}$, $i=1,2, \ldots$. By Lemma [7.1] it is enough to argue that for every $i=1,2, \ldots$ the backward semipath $W_{i}^{b}=\left(\gamma_{x_{i}, t_{i}}(s), s\right)_{s \leq t_{i}}$ is determined by $\mathcal{W}=\left\{W_{i}, i=\right.$ $1,2, \ldots\}$. Let $\overline{\mathcal{W}}$ be the closure of $\mathcal{W}$ in $\mathcal{H}$. It readily follows from the properties of $\breve{\mathcal{W}}$ that $\overline{\mathcal{W}}$ is compact and contains (forward) paths $\eta_{x, t}$, not necessarily unique, starting from each $(x, t) \in \overline{\mathbb{R}}^{2}$, all of which are semipaths of $\breve{\mathcal{W}}$. Fix now $i_{0} \geq 0$ and let

$$
\begin{aligned}
& \mathbb{D}^{+}=\left\{(x, t) \in \overline{\mathbb{R}}^{2}: t \leq t_{i_{0}}, \eta_{x, t}\left(t_{i_{0}}\right) \geq W_{i_{0}}\left(t_{i_{0}}\right) \text { for some } \eta_{x, t} \in \overline{\mathcal{W}}\right\} \\
& \mathbb{D}^{-}=\left\{(x, t) \in \overline{\mathbb{R}}^{2}: t \leq t_{i_{0}}, \eta_{x, t}\left(t_{i_{0}}\right) \leq W_{i_{0}}\left(t_{i_{0}}\right) \text { for some } \eta_{x, t} \in \overline{\mathcal{W}}\right\} \\
& \mathbb{D}=\mathbb{D}^{+} \cap \mathbb{D}^{-}
\end{aligned}
$$


We claim that $\mathbb{D}=W_{i_{0}}^{b}$ (as a subset of $\overline{\mathbb{R}}^{2}$ ). Indeed, given $(y, s) \in W_{i_{0}}^{b}$ with $s<t_{i_{0}}$, if $\left(y_{n}, s_{n}\right)_{n \geq 1}$ approaches $(y, s)$ with $y_{n}>y$ for $n \geq 1$, then, by the compactness of $\overline{\mathcal{W}},\left(\eta_{y_{n}, s_{n}}\right)_{n \geq 1}$ has a limit point $\eta_{y, s}$ in $\overline{\mathcal{W}}$ starting from $(y, s)$, which being a semipath from a path of $\breve{\mathcal{W}}$ does not cross $W_{i_{0}}^{b}$. Thus $(y, s) \in \mathbb{D}^{+}$. With an analogous argument taking $y_{n}<y$, we conclude that $(y, s) \in \mathbb{D}^{-}$, and thus $W_{i_{0}}^{b} \subset \mathbb{D}$.

If $(y, s) \in \mathbb{D} \backslash W_{i_{0}}^{b}$, then there is a semipath $\eta_{y, s}$ of $\breve{\mathcal{W}}$ starting in $(y, s)$ which passes through $\left(x_{i_{0}}, t_{i_{0}}\right)$, and thus a path in $\breve{\mathcal{W}}$ through $\left(x_{i_{0}}, t_{i_{0}}\right)$ other than $\gamma_{x_{i_{0}}, t_{i_{0}}}$, contradicting the hypothesis.

Proof of Lemma 2.3 It suffices to show that there is only one path in $\breve{\mathcal{W}}$ through each point of $\mathcal{D}$, and then invoke Lemma 2.2. Given $\left(x_{0}, t_{0}\right) \in \mathcal{D}$, we have at least one path in $\breve{\mathcal{W}}$ through it, since we already have the semipath $W_{x_{0}, t_{0}}$. If there were two different paths $\gamma$ and $\gamma^{\prime}$ in $\breve{\mathcal{W}}$ through $\left(x_{0}, t_{0}\right)$, then, since $\mathcal{D}$ is dense, there would exist a point $\left(x_{1}, t_{1}\right)$ in $\mathcal{D}$ with $t_{1}<t_{0}$ and $x_{1}$ strictly between $\gamma$ and $\gamma^{\prime}$, and thus, by the noncrossing property, $W_{x_{1}, t_{1}}$ would not be able to avoid $\left(x_{0}, t_{0}\right)$, contradicting the hypothesis that each $W_{x_{i}, t_{i}}$ avoids all other points of $\mathcal{D}$.

Proof of Proposition 3.1]Fix a deterministic countable dense $\mathcal{D} \in \mathbb{R}^{2}$ and let $\mathcal{W}=\mathcal{W}(\mathcal{D})$ be the set of paths of $\overline{\mathcal{W}}$ starting from $\mathcal{D}$. Then $\mathcal{W}$ is almost surely a Brownian web skeleton. To fix notation, we write $\mathcal{D}=\left\{\left(x_{i}, t_{i}\right), i \geq\right.$ $1\}, \mathcal{W}=\left\{\hat{W}_{i}, i \geq 1\right\}$, where $\hat{W}_{i}$ is the path of $\mathcal{W}$ starting from $\left(x_{i}, t_{i}\right)$, $i \geq 1$. We proceed, as in [8, 4] and with the same notation, to define sets of coalescing/reflecting dual paths $\hat{W}_{1}^{b, n}, \hat{W}_{2}^{b, n}, \ldots$ inductively, as follows. Let $B_{j}^{b}, j \geq 1$ be i.i.d. standard Brownian motions which are independent of $\mathcal{W}$, and let $W_{j}^{b}$ be as in Equation 3.2 of [4. For $n>1$, let

$$
\begin{aligned}
& \hat{W}_{1}^{b, n}=C R\left(W_{1}^{b} ; \hat{W}_{1}, \hat{W}_{2}, \ldots, \hat{W}_{n}\right) ; \\
& \hat{W}_{j}^{b, n}=C R\left(W_{j}^{b} ; \hat{W}_{1}, \ldots, \hat{W}_{n}, \hat{W}_{1}^{b, n}, \ldots, \hat{W}_{j-1}^{b, n}\right), 1<j \leq n .
\end{aligned}
$$

By Theorem 8 of [8], $\left\{\hat{W}_{1}, \ldots, \hat{W}_{n}, \hat{W}_{1}^{b, n}, \ldots, \hat{W}_{j}^{b, n}\right\}$ has the same distribution as $\left\{\tilde{W}_{1}, \ldots, \tilde{W}_{n}, \tilde{W}_{1}^{b}, \ldots, \tilde{W}_{j}^{b}\right\}$ defined in Equations (3.3)-(3.5) of [4. Since the latter converges weakly to the DBW, then so does the former.

We claim that for each $i \geq 1, \Gamma_{i}:=\left[\left\{\hat{W}_{i}^{b, n}, 1<n \leq m\right\}, m>1\right]$ is tight. Thus by Proposition B.4 of [3], $\left\{\hat{W}_{i}^{b, n}, 1<n<\infty\right\}$ is a.s. precompact.

We argue now that $\hat{W}_{i}^{b, n} \rightarrow \hat{W}_{i}^{b}$ almost surely as $n \rightarrow \infty$ for all $i \geq 1$. If that were not the case, by the above precompactness, there would exist two 
different subsequential limits for $\left\{\hat{W}_{i}^{b, n}, 1<n<\infty\right\}$, say $\check{W}_{i}^{b}$ and $\breve{W}_{i}^{b}$, both backward paths starting at $\left(x_{i}, t_{i}\right)$ and not crossing $\mathcal{W}$. There must thus exist $\left(x_{i^{\prime}}, t_{i^{\prime}}\right) \in \mathcal{D}$ with $t_{i^{\prime}}<t_{i}$ and $x_{i^{\prime}}$ strictly between $\check{W}_{i}^{b}\left(t_{i^{\prime}}\right)$ and $\breve{W}_{i}^{b}\left(t_{i^{\prime}}\right)$. This and noncrossing imply that $W_{i^{\prime}}\left(t_{i}\right)=x_{i}$, which is a null event.

We thus have that for all $k \geq 1$,

$$
\left\{\hat{W}_{1}, \ldots, \hat{W}_{k}, \hat{W}_{1}^{b, n}, \ldots, \hat{W}_{k}^{b, n}\right\} \rightarrow\left\{\hat{W}_{1}, \ldots, \hat{W}_{k}, \hat{W}_{1}^{b}, \ldots, \hat{W}_{k}^{b}\right\} \text { as } n \rightarrow \infty .
$$

By one of the above arguments, $\left\{\hat{W}_{1}, \ldots, \hat{W}_{k}, \hat{W}_{1}^{b}, \ldots, \hat{W}_{k}^{b}\right\}$ has the same distribution as $\left\{\tilde{W}_{1}, \ldots, \tilde{W}_{n}, \tilde{W}_{1}^{b}, \ldots, \tilde{W}_{j}^{b}\right\}$ defined in Equations (3.3)-(3.5) of 4 . It follows that

$$
\left\{\hat{W}_{1}, \ldots, \hat{W}_{k}, \hat{W}_{1}^{b}, \ldots, \hat{W}_{k}^{b}\right\} \rightarrow\left(\hat{\mathcal{W}}, \hat{\mathcal{W}}^{b}\right) \text { as } k \rightarrow \infty
$$

where $\left(\hat{\mathcal{W}}, \hat{\mathcal{W}}^{b}\right)$ is a standard DBW and the convergence is in $\mathcal{H} \times \mathcal{H}^{b}$. Clearly $\hat{\mathcal{W}}=\overline{\mathcal{W}}$.

It remains to justify the above tightness claim. We verify the tightness condition $\left(T_{1}\right)$ from Appendix B of 3 for $\Gamma_{i}$. The reasoning goes by a blocking argument which is a entirely similar to the one used for Proposition B.3 in that reference. To facilitate the adaptation of that argument, let $\mathcal{X}_{m}^{b}=\left\{\hat{W}_{i}^{b, n}, 1<n \leq m\right\}$ and $\mathcal{X}_{m}=\left\{\hat{W}_{1}, \ldots, \hat{W}_{m}\right\}$. We then have that the paths of $\mathcal{X}_{m}^{b}$ do not cross those of $\mathcal{X}_{m}$, and the latter satisfies condition $\left(I_{1}^{\prime}\right)$ of that proposition.

The blocking argument of the proof of Proposition B.3 in 3 consists of subdividing the intervals $\left[x_{0}-u / 2, x_{0}-u / 4\right] \times\left\{t_{0}\right\}$ and $\left[x_{0}+u / 2, x_{0}+u / 4\right] \times$ $\left\{t_{0}\right\}$ into subintervals whose lengths are of order $\sqrt{t}$, and then arguing that the limsup $\operatorname{su}_{m \rightarrow \infty}$ of the probability of either not having a forward crossing by any of the paths of $\mathcal{X}_{m}$ of any of the rectangles to the right of $\left(x_{0}, t_{0}\right)$ with height $2 t$ and having the respective subintervals as base, or of not having a forward crossing by any of the paths of $\mathcal{X}_{m}$ of any of the rectangles to the left of $\left(x_{0}, t_{0}\right)$ with height $2 t$ having the respective subintervals as base is $o\left(t^{-1}\right)$. In the present case the same of course holds true, and as soon as there is a blocking at each side, there cannot be a horizontal crossing by paths in $\mathcal{X}_{m}^{b}$. Condition $\left(T_{1}\right)$ is thus verified and tightness of $\Gamma_{i}$ follows.

\section{References}

[1] R. Arratia, Coalescing Brownian motions and the voter model on $\mathbb{Z}$, Unpublished partial manuscript (circa 1981), available from rarratia@math.usc.edu. 
[2] L. R. G. Fontes, M. Isopi, C. M. Newman, K. Ravishankar, The Brownian web, Proc. Natl. Acad. Sci. USA 99, 15888-15893 (2002).

[3] L. R. G. Fontes, M. Isopi, C. M. Newman, K. Ravishankar, The Brownian web: characterization and convergence, Ann. Probability 32, 2857-2883 (2004); arXiv: math.PR/0304119.

[4] L. R. G. Fontes, M. Isopi, C. M. Newman, K. Ravishankar, Coarsening, Nucleation, and the Marked Brownian Web, Ann. Inst. H. Poincaré Probab. Statist., in press.

[5] T. E. Harris, Coalescing and noncoalescing flows in $\mathbb{R}^{1}$, Stoch. Proc. and their Appl. 17, 187-210 (1984).

[6] V. V. Piterbarg, Expansions and contractions of stochastic flows, Ph. D. dissertation, Univ. Southern California (1997).

[7] V. V. Piterbarg, Expansions and contractions of isotropic stochastic flows of homeomorphisms, Ann. Probability 26, 479499 (1998).

[8] F. Soucaliuc, B. Tóth, W. Werner, Reflection and coalescence between independent one-dimensional Brownian paths, Ann. Inst. H. Poincaré Probab. Statist. 36, 509-545 (2000).

[9] B. Tóth, W. Werner, The true self-repelling motion, Probab. Theory Related Fields 111, 375-452 (1998). 